\title{
Chromosome painting in the manatee supports Afrotheria and
} Paenungulata

\author{
Margaret E Kellogg1, Sandra Burkett ${ }^{2}$, Thomas R Dennis ${ }^{3}$, Gary Stone ${ }^{2}$, \\ Brian A Gray ${ }^{3}$, Peter M McGuire ${ }^{4}$, Roberto T Zori ${ }^{3}$ and Roscoe Stanyon*5
}

\begin{abstract}
Address: ${ }^{1}$ College of Veterinary Medicine, University of Florida, PO BOX 100245, Gainesville, FL 32610-0245, USA, ${ }^{2}$ Comparative Molecular Cytogenetics Core, National Cancer Institute, Frederick, MD 21702, USA, ${ }^{3}$ Department of Pediatrics, Division of Genetics, University of Florida, PO Box 100296, UFHSC, Gainesville, FL 32610, USA, "4Department of Biochemistry and Molecular Biology, University of Florida, PO Box 100245 , College of Medicine, Gainesville, FL 32610, USA and ${ }^{5}$ Department of Animal Biology and Genetics, University of Florence, Florence, Italy, Via del Proconsolo 12, 50122 Florence, Italy (formerly at NCI, Frederick)

Email: Margaret E Kellogg - kelloggm@gmail.com; Sandra Burkett - sburkett@ncifcrf.gov; Thomas R Dennis - tdennis@tgen.org; Gary Stone - gstone@ncifcrf.gov; Brian A Gray - grayb@pathology.ufl.edu; Peter M McGuire - pmcguire@biochem.med.ufl.edu; Roberto T Zori - zorirt@peds.ufl.edu; Roscoe Stanyon* - roscoe.stanyon@unifi.it

* Corresponding author
\end{abstract}

Published: 23 january 2007

BMC Evolutionary Biology 2007, 7:6 doi:10.1/86/147/-2/48-7-6
Received: 18 October 2006

Accepted: 23 January 2007

This article is available from: http://www.biomedcentral.com//47/-2/48/7/6

(C) 2007 Kellogg et al; licensee BioMed Central Ltd.

This is an Open Access article distributed under the terms of the Creative Commons Attribution License (http://creativecommons.org/licenses/by/2.0), which permits unrestricted use, distribution, and reproduction in any medium, provided the original work is properly cited.

\begin{abstract}
Background: Sirenia (manatees, dugongs and Stellar's sea cow) have no evolutionary relationship with other marine mammals, despite similarities in adaptations and body shape. Recent phylogenomic results place Sirenia in Afrotheria and with elephants and rock hyraxes in Paenungulata. Sirenia and Hyracoidea are the two afrotherian orders as yet unstudied by comparative molecular cytogenetics. Here we report on the chromosome painting of the Florida manatee.
\end{abstract}

Results: The human autosomal and $X$ chromosome paints delimited a total of 44 homologous segments in the manatee genome. The synteny of nine of the 22 human autosomal chromosomes $(4,5,6,9,11,14,17,18$ and 20$)$ and the $X$ chromosome were found intact in the manatee. The syntenies of other human chromosomes were disrupted in the manatee genome into two to five segments. The hybridization pattern revealed that 20 ( 15 unique) associations of human chromosome segments are found in the manatee genome: $1 / 15,1 / 19,2 / 3$ (twice), 3/7 (twice), 3/13, 3/2I, 5/21, 7/16, 8/22, 10/12 (twice), II/20, 12/22 (three times), 14/15, 16/19 and 18/19.

Conclusion: There are five derived chromosome traits that strongly link elephants with manatees in Tethytheria and give implicit support to Paenungulata: the associations $2 / 3,3 / 13,8 / 22,18 / 19$ and the loss of the ancestral eutherian $4 / 8$ association. It would be useful to test these conclusions with chromosome painting in hyraxes. The manatee chromosome painting data confirm that the associations I/19 and 5/2I phylogenetically link afrotherian species and show that Afrotheria is a natural clade. The association $10 / / 2 / 22$ is also ubiquitous in Afrotheria (clade I), present in Laurasiatheria (clade IV), only partially present in Xenarthra (I0/I2, clade II) and absent in Euarchontoglires (clade III). If Afrotheria is basal to eutherians, this association could be part of the ancestral eutherian karyotype. If afrotherians are not at the root of the eutherian tree, then the $10 /$ 12/22 association could be one of a suite of derived associations linking afrotherian taxa. 


\section{Background}

Recently the molecular based approaches of super-ordinal grouping of extant eutherians (Afrotheria, Euarchontoglires, Laurasiatheria and Xenarthra) has gained popularity [1-3]. However, one of the four proposed super-orders, Afrotheria, is controversial because it unites morphologically distinct species of African placentals (golden moles, tenrecs, otter shrews, elephant shrews, aardvarks, hyraxes, elephants and sirenians). Within Afrotheria, sirenians, elephants and hyraxes form a clade called Paenungulata. There is little morphological or paleontological evidence that provides support for Afrotheria [4]. A movable snout was hypothesized as a synapomorphic trait, but this feature is apparently not homologous across different afrotherian lineages[5]. More recently, it was proposed that aspects of placentation could provide a synapomorphy for this assemblage $[6,7]$. Some outstanding issues in higher eutherian phylogenomics include the exact root of the placental tree, the relationships within the super-ordinal clade Laurasiatheria (moles, hedgehogs, shrews, bats, cetaceans, ungulates, pangolins and carnivores), and resolving the trichotomy of sirenians, elephants and hyraxes [8].

Sirenia and Hyracoidea are the two afrotherian orders remaining to be investigated with molecular cytogenetic techniques. In this paper, the chromosome painting of the Florida manatee (Trichechus manatus latirostris) is reported. These data should be a valuable addition to our understanding of afrotherian relationships and the eutherian ancestral karyotype.

\section{The Florida manatee}

The endangered Florida manatee is a subspecies of the West Indian manatee (Trichechus manatus) in the order Sirenia. Sirenians are often considered phylogenetic outliers. Despite similarities in adaptations, habitat, and body shape, they have no evolutionary relationship with the other orders of marine mammals. Extant sirenians are the only herbivorous marine mammals and live in fresh, brackish or marine habitats dispersed along tropical and subtropical environments.

\section{Previous cytogenetic reports on manatees}

Solid stained chromosome studies were completed on a limited number of individual manatees, establishing the chromosome number as $2 \mathrm{~N}=48$ for the Florida manatee $[9,10]$ and $2 \mathrm{~N}=56$ for the Amazonian manatee (Trichechus inunguis) [11]. Following solid staining, chromosome-banding procedures allowed for the identification of individual chromosome regions. Giemsa and trypsin staining, or GTG-banding, was used to create karyotypes and ideograms for the Florida manatee [12] and the Amazonian manatee [13].
Comparisons of chromosome painting data provide an independent test of the contrasting hypotheses on mammalian evolution and phylogeny. The research presented here clarifies the phylogenetic position of the manatee and tests the validity of the radical taxonomic assemblage known as Afrotheria. The results are then compared to other chromosome painting data in Afrotheria. In light of the findings, the relationships within Afrotheria and the alternative organizations of the ancestral eutherian karyotype are assessed.

\section{Results}

Examples of human chromosome paints (HSA) hybridized to manatee (TMA) metaphase chromosomes are shown in Figure 1. Synteny was found intact in nine $(4,5$, $6,9,11,14,17,18$ and 20) of the 22 human autosomal and $\mathrm{X}$ chromosomes (Figure 2). Two hybridization signals were evident on separate manatee chromosomes for ten human chromosomes $(1,7,8,10,12,13,15,16,21$ and 22). The human 19 paint hybridized to three TMA chromosomes $(2,12$ and 14). Human chromosomes 2 and 3 were highly fragmented in the manatee genome and painted four and five chromosomes, respectively (Table 1 ). Due to the small signals involved and the quality of the metaphases, it was more difficult to assign the hybridization pattern for these two chromosomes. Human chromosome paint 12 provided three signals on TMA 7, most likely due to an inversion. Chromosome paints with pericentromeric signals on both arms of the same chromosome were considered as one signal. Centromere areas on the manatee karyotype were not hybridized. The Y chromosome was the only human probe that failed to provide a signal in the manatee. Altogether, the human autosomal chromosome paints and the X chromosome paint delimited a total of 44 homologous segments in the manatee genome. Human chromosome paints hybridized to 20 (15 unique) segments in the manatee genome: $1 / 15,1 /$ 19, 2/3 (twice), 3/7 (thrice), 3/13, 3/21, 5/21, 7/16, 8/22, $10 / 12$ (twice), $11 / 20,12 / 22$ (thrice), 14/15, 16/19 and $18 / 19$.

\section{Discussion}

The painting map of the manatee genome was compared with results published on other Afrotheria taxa: aardvark, elephant, elephant shrew and golden mole [14-17]. An assessment of the associations found in each taxa are shown in Table 1. All species have eight associations in common $(1 / 19,3 / 21,5 / 21,7 / 16,10 / 12,12 / 22,14 / 15$ and 16/19). Five of these associations are considered ancestral to all eutherians by most proposals $(3 / 21,7 / 16$, $12 / 22$ twice, $14 / 15$ and 16/19). It appears that the associations $1 / 19$ and $5 / 21$ can be used to link afrotherian species $[14-16,18]$. These associations provide cytogenetic support, in agreement with molecular studies, that Afrotheria is a natural clade. 

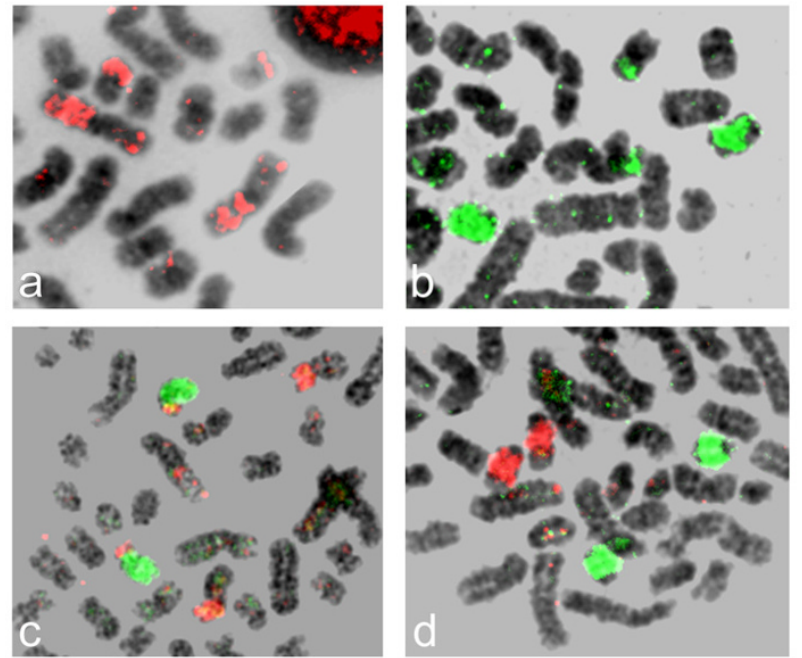

\section{Figure I}

Examples of hybridizations in the manatee a) human 12, b) human 13, c) human 14 in green and 15 in red d) human 17 in green and 18 in red.

New chromosome painting data in Xenarthra (anteaters, sloths and armadillos) are also informative towards the ancestral eutherian karyotype. Of the four species studied, Tamandua tetradactyla, Choloepus didactylus, C. hoffmanii and Dasypus novemcinctus $[18,19]$, only the anteater has a $1 / 19$ association. It is not likely that this association is homologous to Afrotheria, because the anteater has the most highly rearranged karyotype known in Xenarthra [18].

The manatee data indicate that the association 10/12/22 is most likely ubiquitous throughout Afrotheria. A combination HSA $10 p / 12 p / 22 q$ and a single HSA10q were found in the aardvark and elephant karyotypes $[14,17]$. An apparently identical association was later found in the elephant shrew and golden mole [15]. The question is, whether this association is a third cytogenetic landmark for the Afrotheria clade, or instead should be considered part of the ancestral eutherian karyotype.

The entire 10/12/22 association appears to be present in clades I, Afrotheria, and IV, Laurasiatheria, only partially present in clade II, Xenarthra (10/12), and absent in clade III, Euarchontoglires (primates, rabbits, rodents, tree shrews and flying lemurs). Carnivores have a homologous 10/12/22 association to Afrotheria, as demonstrated by reciprocal chromosome painting [20,21]. Eulipotyphla (shrews, solenodons, moles, hedgehogs, and Nesophontes) also have the 10/12/22 association [19,22]. Chromosome painting data in Xenarthra show that a 10/12 association is present in the armadillo (D. novemcinctus) [18]. To date, the 10/12 association has been found in three of the four eutherian mammal clades. Yet, there is no reciprocal painting in Xenarthra to prove that the 10/12 association is truly homologous to that found in Afrotheria. Several hypotheses can be developed with different implications if Afrotheria or Xenarthra is considered basal. If Afrotheria is basal, the occurrence of $10 / 12 / 22$ in clades I and IV would suggest that this association is part of the ancestral eutherian karyotype with a subsequent, independent loss in clades II and III. The occurrence of the 10/12/22 association clades I and IV, could be considered a phylogenetic link. Alternatively, the association could have been independently acquired in the two clades. If Xenarthra is basal, this association could have originated in Afrotheria and was then lost in clade III.

Association 3/13 was found in the manatee, elephant and elephant shrew. However, there are no reciprocal painting data between human and manatee or human and elephant shrew. Therefore, it is not possible to confirm that the $3 / 13$ association is homologous (involves the same segments of both chromosomes 3 and 13). In view of the afrotherian molecular data, this association was independently derived in the Macroscelidae (elephant shrews) and Paenungulata phylogenetic lineages [8].

\section{Support for the Tethytheria and Paenungulata assemblage} Before the advent of molecular studies, some morphologists placed sirenians, elephants and hyraxes under Ungulata. Elephants and sirenians were grouped together in Tethytheria, while hyraxes were placed in Phenacodonta along with perissodactyls [23]. Results in molecular studies are inconsistent and fail to resolve the Paenungulate trifurcation [8] and some data do not support Tethytheria [2,24-26]. Mitochondrial genome analyses do support Tethytheria, but exclude Hyracoidea [1]. SINE insertion data produced incongruent phylogenetic relationships within Paenungulata, most likely due to a rapid divergence from a highly polymorphic last common ancestor [27].

The chromosome mapping data strongly support Tethytheria (sirenians and elephants) and implies support for the clade Paenungulata (Sirenia, Proboscidea, and Hyracoidea). There appear to be four derived associations linking elephants with manatees: $2 / 3,3 / 13,8 / 22$ and $18 / 19$. HSA $4 / 8 \mathrm{p}$ was not present in the manatee and may represent a derived trait of Paenungulata. Both publications on the elephant indicate that this association is also lacking $[14,17]$. It is possible that the $4 / 8$ association went undetected in our study, as well as in elephants. Although, the widespread occurrence of the $4 / 8$ association in all mammals, outside of elephants and most primates, lends credence to its inclusion in the ancestral eutherian karyotype. 

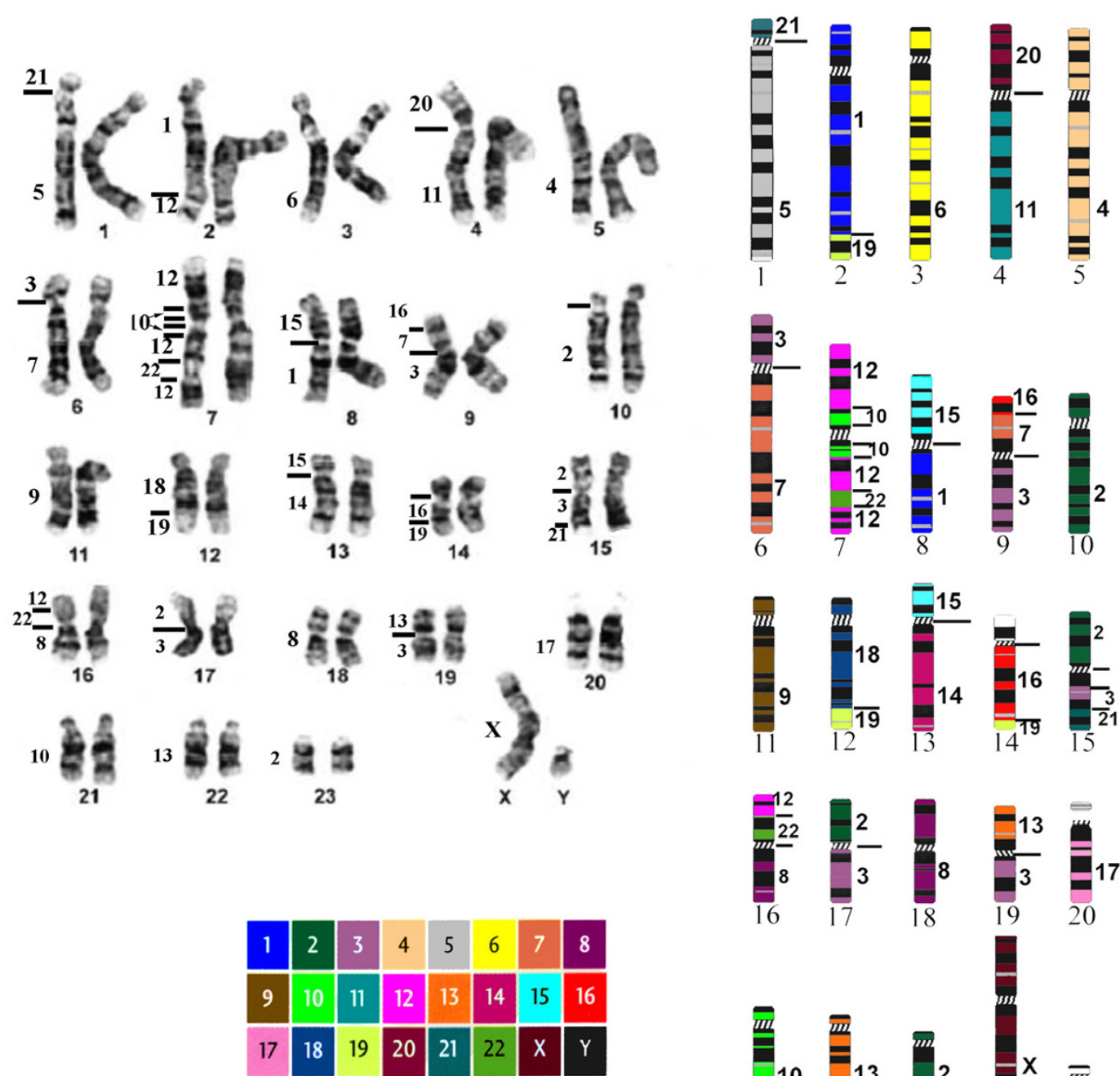

human chromosome color key
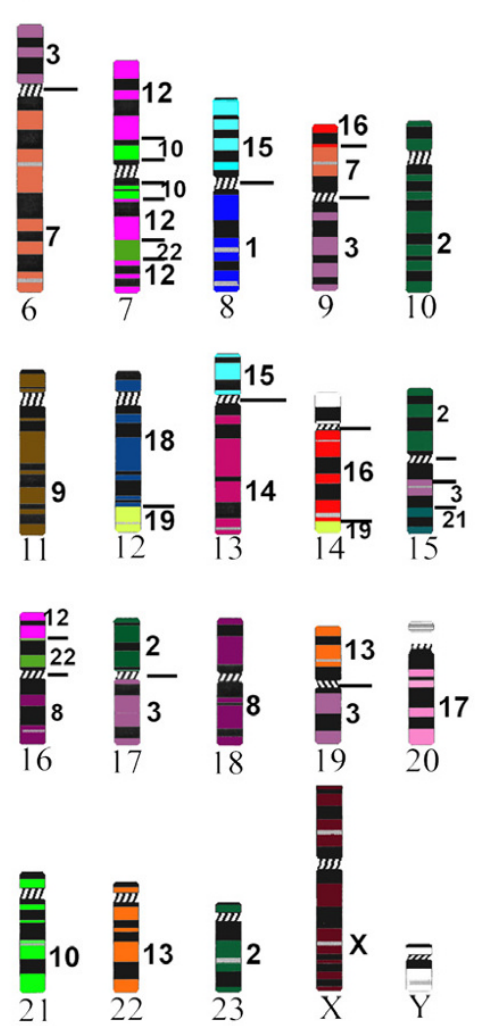

Figure 2

The karyotype of the manatee is shown to the left and the color coded idiogram to the right (modified from Gray et al. 2002). Manatee chromosomes are numbered below and human chromosome homology is shown laterally. 
Table I:

\begin{tabular}{lcccccccccccccccccccccccccccc}
\hline & $2 n$ & 1 & 2 & 3 & 4 & 5 & 6 & 7 & 8 & 9 & 10 & 11 & 12 & 13 & 14 & 15 & 16 & 17 & 18 & 19 & 20 & 21 & 22 \\
\hline AEK & 48 & 1 & 2 & 1 & 1 & 1 & 1 & 2 & 2 & 1 & 2 & 1 & 2 & 1 & 1 & 1 & 2 & 1 & 1 & 2 & 1 & 1 & 2 \\
manatee & 48 & 2 & 4 & 5 & 1 & 1 & 1 & 2 & 2 & 1 & 2 & 1 & $2(4)$ & 2 & 1 & 2 & 2 & 1 & 1 & 3 & 1 & 2 & 2 \\
golden mole & 30 & 1 & 2 & 1 & 1 & 1 & 1 & 2 & 2 & 1 & 2 & 1 & 2 & 1 & 1 & 1 & 2 & 1 & 1 & 2 & 1 & 1 & 2 \\
elephant shrew & 26 & 1 & 2 & 4 & 1 & 1 & 1 & 2 & 2 & 1 & 2 & 1 & 2 & 1 & 1 & 1 & $2(3)$ & 1 & 1 & $2(3)$ & 1 & 1 & 2 \\
aardvark & 20 & 1 & 2 & 1 & 1 & 1 & 1 & 2 & 2 & 1 & 2 & 1 & 2 & 1 & 1 & 1 & 2 & 1 & 1 & 2 & 1 & 1 & 2 \\
elephant & 56 & 4 & 4 & 5 & 3 & 1 & 1 & 2 & 2 & 1 & 2 & 3 & 2 & 2 & 1 & 2 & 2 & 1 & 1 & 3 & 1 & 2 & 2 \\
\hline
\end{tabular}

Number of segments homologous to human chromosome found in Afrotheria species. The taxa in the first, left column: AEK = ancestral eutherian karyotype $[14,16,40]$. The second column list the $2 \mathrm{n}$, diploid numbers for each species and the remaining columns refer to signals found for each human chromosome. The number in brackets refers to higher number of hybridization signals due to pericentric inversions.

It would be useful to test these hypotheses with rock hyrax chromosome painting data.

\section{Branching order in Afrotheria}

The branching order within Afrotheria has not reached a consensus. Some authors have viewed Macroscelidae, the elephant shrews, as the most basal and early divergent order within Afrotheria [2,28]. However, Murphy et al. (2001) placed the triumvirate of sirenians, elephants and hyraxes (Paenungulata) as basal, verified by additional molecular data $[1,3,29]$. It is difficult to determine which order is most basal because sirenians and elephants, like other afrotherian species, have fairly derived karyotypes.

According to Robinson et al. (2003), associations 2/8, 3 / 20 and 10/17 link elephant shrews, golden moles/tenrecs and aardvarks. Only the association $2 / 8$ is present in all three. Recently, the association $2 / 8$ was also found in anteater (T. tetradactyla), sloth (Choloepus didactylus) and pangolin (Manis javanica) [18,19]. Associations 3/20 and 10/ 17 are lacking in golden moles/tenrecs. Murphy et al. (2004) proposed that the associations 3/20 and 10/17 were probably lost in golden moles/tenrecs. No reciprocal painting was done in elephant shrews or golden moles/ tenrecs and it is therefore unknown if these associations are actually homologous. There is weak cytogenetic evidence linking elephant shrews and golden moles/tenrecs. An alternate hypothesis might be a sister relationship between elephant shrews and aardvarks. Perhaps a rapid divergence in elephant shrews, golden moles/tenrecs and aardvarks also resulted in limited phylogenetic signals for these chromosome associations.

\section{The root of the Eutherian tree}

Although the super-order assemblies appear well established, the most basal position on the eutherian tree has not been determined with certainty $[2,25,30]$. Afrotheria and Xenarthra are the two oldest eutherian clades and probably emerged from the Southern Hemisphere in excess of 100 million years ago [31,32]. Molecular dating and biogeography have provided evidence that crowngroup Eutheria may have their most recent common ancestry in the Southern Hemisphere (Gondwana) [32]. The other two clades (Laurasiatheria and Euarchontoglires) can be grouped as Boreoeutheria [33].

There are currently three hypotheses for the root of the eutherian tree. Most discussions from molecular studies place emphasis on either Afrotheria or Xenarthra as the most basal clade $[25,34]$. A third hypothesis states that the ancestral eutherian karyotype is a combination of both clades. This hypothesis cannot be completely ruled out and is preferred in some studies [35,36]. However, the suite of derived chromosomal associations found in all studied Afrotheria argues against the hypothesis that a combination of the two clades is basal to the eutherians.

Recently, a report on retroelements gives support for the hypothesis that Xenarthra is the sister group to all other placentals [37]. Indeed, new cytogenetic comparisons show that the proposed ancestral eutherian karyotype is essentially conserved in Xenarthra, specifically in the twotoed sloth (Choloepus hoffmanii) [16]. These two studies should be given attention because both take into consideration rare genomic events in which convergence is particularly limited. The conserved xenarthran karyotype may well be indicative of their phylogenomic position among eutherians. However, an essential point is that all reconstructions of the ancestral eutherian karyotype are preliminary until a relevant outgroup is studied with chromosome painting. A taxonomically rich array of species supported by appropriate out-groups is vital to the reconstruction of mammalian genome evolution. The deficiency of comparative chromosome painting data between eutherians and marsupials is a severe limitation on attempts to delineate the mammalian ancestral genome. The analyses of other afrotherians, xenarthrans and marsupials may clarify these unresolved questions.

\section{Conclusion}

The chromosome painting data presented here leave little doubt that Tethytheria is a clade within Afrotheria and implies support for the Paenungulata assemblage. Recent retroposon data also confirmed Paenungulata, but could 
not resolve the phylogenetic relationships among elephants, sirenians and hyraxes [27]. It is generally appreciated that characters with high evolutionary rates provide good phylogenetic resolution. Afrotherian karyotypes demonstrate high rates of chromosome evolution and numerous derived inter-chromosomal rearrangements link elephants and manatees. It is therefore likely that additional chromosome painting in rock hyraxes could shed light on the divergence sequence and resolve the Paenungulata trichotomy.

\section{Methods}

Chromosome preparations of a male Florida manatee (Trichechus manatus latirostris, TMA) were obtained from peripheral blood mononuclear cells (PBMCs) and primary fibroblast cartilage cell culture. Cells were cultured in RPMI 1640 (Hyclone) supplemented with 20\% fetal bovine serum (FBS), L-glutamine $(0.01 \%)$ and gentamicin $(25 \mu \mathrm{g} / \mathrm{ml})$. PBMCs were incubated in-vivo using phytohemagglutinin (PHA, $0.25 \mathrm{mg} / \mathrm{mL}$ ) as a mitotic stimulant for 72 to $96 \mathrm{hr}$ at $36^{\circ} \mathrm{C}$ in $5 \%$ carbon dioxide, $95 \%$ air and $100 \%$ relative humidity. Routine procedures were used for chromosome preparations. We followed the chromosome nomenclature as previously published [12] pairing and grouping chromosomes by banding patterns, relative lengths and morphology.

Human chromosome paints were obtained as previously described by chromosome flow sorting followed by degenerate oligonucleotide primed PCR amplification [38,39]. Paints were labeled with either biotin-dUTP, digoxigen-dUTP (both from Roche Applied Science) or Spectrum Orange-dUTP (Vysis).

Interspecific in-situ hybridizations of Florida manatee chromosomes with human probes were performed with 300 to $500 \mathrm{ng}$ of each biotin-labeled probe, $10 \mu \mathrm{g}$ of human Cot-1 DNA and $5 \mu \mathrm{g}$ of ssDNA. The mixture was precipitated and dissolved in 13-15 $\mu$ l of hybridization mixture (formamide 50\%, dextran sulfate 10\%, $2 \times$ SSC). Direct labeling with Spectrum Orange followed a Nick Translation protocol (Vysis) using $1 \mu \mathrm{g}$ of each amplified human DNA probe, $0.2 \mathrm{mM}$ Spectrum Orange and $25 \mu \mathrm{g}$ each of human and manatee Cot-1 DNA (Applied Genetics Laboratories, Inc.). The mixture was precipitated and dissolved in $10 \mu \mathrm{l}$ distilled water. Approximately $300 \mathrm{ng}$ of probe from this mixture were dissolved in $10.5 \mu \mathrm{l}$ Hybrizol VII (Q-BIOgene) and $0.75 \mu \mathrm{g}$ each of human and manatee Cot-1 DNA.

The labeled probe mixture was denatured at $80^{\circ} \mathrm{C}$ for 10 min and reannealed at $37^{\circ} \mathrm{C}$ for 90 min before hybridization. Slides were aged at $37^{\circ} \mathrm{C}$ for $30 \mathrm{~min}$ followed by dehydration in a room temperature $70,80,90$, and $100 \%$ ethanol series. The DNA was denatured in $70 \%$ formamide $/ 2 \times \mathrm{SSC}$, at $65^{\circ} \mathrm{C}$ for $90-120 \mathrm{~s}$, and quenched in an ice-cold ethanol series. Hybridization was carried out in a humidity chamber at $37^{\circ} \mathrm{C}$ for five days. Post-hybridization washes followed standard procedures at $40^{\circ} \mathrm{C}$. Biotin detection was performed with avidin-conjugated FITC (Vector) for $45 \mathrm{~min}$ at $37^{\circ} \mathrm{C}$. Counterstaining was performed with DAPI $(0.8 \mathrm{ng} / \mu \mathrm{l})$ for $10 \mathrm{~min}$ and the slides were mounted with antifade $(100 \mathrm{mg}$ p-phenylenediamine in $80 \mathrm{ml}$ glycerine, $20 \mathrm{ml}$ PBS, $\mathrm{pH}$ 8).

Analyses were performed under a Zeiss Axiophot 2 or Axioskop fluorescence microscope coupled with a CCD camera (Photometrics), and images were captured with the Smart Capture software (Digital Scientific Inc.).

\section{Authors' contributions}

$\mathrm{RS}$ and RZ conceived and designed the experiments. MK, $\mathrm{SB}, \mathrm{TD}$ and RS performed the experiments. BG, MK, RZ, GS and PM prepared and contributed reagents/cell cultures/analysis tools. RS, MK and SB analysed the data. RS, MK and PM wrote the paper. All author read and approved the final manuscript.

\section{Acknowledgements}

RS was supported by a grant "Mobility of Italian and foreign researchers residing abroad" from MIUR (Ministero Italiano della Universita' e della Ricerca). The U.S. Geological Survey and the University of Florida College of Veterinary Medicine provided support to MK. Samples of manatee tissue were collected under the U.S. Fish and Wildlife Service federal research

Table 2:

\begin{tabular}{|c|c|c|c|c|c|c|c|c|c|c|c|c|c|c|c|c|c|}
\hline & $1 / 19$ & $2 / 3$ & $2 / 8$ & $3 / 13$ & $3 / 20$ & $3 / 21$ & $4 / 8$ & $5 / 21$ & $7 / 16$ & $8 / 22$ & $10 / 12$ & $10 / 17$ & $12 / 22$ & $14 / 15$ & $16 / 19$ & $18 / 19$ & total \\
\hline AEK & & & & & & $x$ & $x$ & & $x$ & & $?$ & & $x$ & $x$ & $x$ & & $6-7$ \\
\hline elephant shrew & $x$ & & $x$ & $x$ & $x$ & $x$ & $x$ & $x$ & $x$ & & $x$ & $x$ & $x$ & $x$ & $x$ & & 21 \\
\hline golden mole & $x$ & & $x$ & & & $x$ & $x$ & $x$ & $x$ & & $x$ & & $x$ & $x$ & $x$ & & 14 \\
\hline Aardvark & $x$ & & $x$ & & $x$ & $x$ & $x$ & $\mathrm{X}$ & $x$ & & $x$ & $x$ & $x$ & $x$ & $x$ & & 20 \\
\hline Elephant & $x$ & $x$ & & $x$ & & $x$ & & $x$ & $x$ & $x$ & $x$ & & $x$ & $x$ & $x$ & $x$ & 17 \\
\hline Manatee & $x$ & $x$ & & $x$ & & $x$ & & $x$ & $x$ & $x$ & $x$ & & $x$ & $x$ & $x$ & $x$ & 15 \\
\hline
\end{tabular}

Chromosomal associations found in two or more Afrotheria taxa. AEK = ancestral eutherian karyotype $[14,16,40]$. Associations were counted only once to derive the total. 
permit MA79I72I issued to the USGS, Sirenia Project. Many thanks for the expertise and support of the UF Cytogenetics Laboratory staff.

\section{References}

I. Murata Y, Nikaido M, Sasaki T, Cao Y, Fukumoto Y, Hasegawa M, Okada N: Afrotherian phylogeny as inferred from complete mitochondrial genomes. Mol Phylogenet Evol 2003, 28(2):253-260.

2. Murphy WJ, Eizirik E, O'Brien SJ, Madsen O, Scally M, Douady CJ, Teel ing E, Ryder OA, Stanhope MJ, de Jong WW, Springer MS: Resolution of the early placental mammal radiation using Bayesian phylogenetics. Science 200I, 294(5550):2348-235I.

3. Springer MS, Murphy WJ, Eizirik E, O'Brien SJ: Placental mamma diversification and the Cretaceous-Tertiary boundary. Proc Natl Acad Sci U S A 2003, 100(3): I056-106I.

4. Stanhope MJ, Madsen O, Waddell VG, Cleven GC, de Jong WW Springer MS: Highly congruent molecular support for a diverse superordinal clade of endemic African mammals. Mol Phylo genet Evol 1998, 9(3):501-508.

5. Whidden HP: Extrinsic snout musculature in Afrotheria and Lipotyphla. Journal of Mammalian Evolution 2002, 9( I/2): | 6 | - I 84.

6. Carter AM, Blankenship TN, Enders AC, Vogel P: The fetal membranes of the otter shrews and a synapomorphy for afrotheria. Placenta 2006, 27(2-3):258-268.

7. Carter AM, Enders AC, Kunzle H, Oduor-Okelo D, Vogel P: Placentation in species of phylogenetic importance: the Afrotheria. Anim Reprod Sci 2004, 82-83:35-48.

8. Murphy WJ, Pevzner PA, O'Brien SJ: Mammalian phylogenomics comes of age. Trends Genet 2004, 20(I 2):631-639.

9. White JR, Harkness DR, Isaacks RE, Duffield DA: Some studies on blood of the Florida manatee, Trichechus manatus latirostris. Comp Biochem Physiol A 1976, 55(4A):4I3-4I7.

10. White JR, Harkness DR, Isaacks RE, Duffield DA: Trichechus manatus latirostris (manatee). Order: Sirenia. Family Trichechidae. In An atlas of mammalian chromosomes Edited by: Hsu TC, Benirschke K. New York, Springer-Verlag; 1977:496.

II. Loughman W, Frye F, Herald E: The chromosomes of a male manatee. International Zoo Yearbook 1970, 10:151-152.

12. Gray BA, Zori RT, McGuire PM, Bonde RK: A first generation ideogram for the Florida manatee (Trichechus manatus latirostris) based on multiple chromosome banding techniques. Hereditas 2002, I37:21 5-223.

13. Assis MFL, Best RC, Yonenagayassuda Y: Cytogenetic study of Trichechus inunguis (Amazonian manatee). Braz J Genet 1988, I I:4 I-50.

14. Fronicke L, Wienberg J, Stone G, Adams L, Stanyon R: Towards the delineation of the ancestral eutherian genome organization: comparative genome maps of human and the African elephant (Loxodonta africana) generated by chromosome paint ing. Proc Biol Sci 2003, 270( I 522): | 33|-|340.

15. Robinson T], Fu B, Ferguson-Smith MA, Yang F: Cross-species chromosome painting in the golden mole and elephant-shrew: support for the mammalian clades Afrotheria and Afroinsectiphillia but not Afroinsectivora. Proc Biol Sci 2004 27I(1547): 1477-1484.

16. Svartman M, Stone G, Page JE, Stanyon R: A chromosome painting test of the basal eutherian karyotype. Chromosome Res 2004 I 2(I):45-53.

17. Yang F, Alkalaeva EZ, Perelman PL, Pardini AT, Harrison WR, O'Brien PC, Fu B, Graphodatsky AS, Ferguson-Smith MA, Robinson TJ: Reciprocal chromosome painting among human, aardvark, and elephant (superorder Afrotheria) reveals the likely eutherian ancestral karyotype. Proc Natl Acad Sci U S A 2003, I00(3): 1062-1066

18. Svartman M, Stone G, Stanyon R: The ancestral eutherian karyotype is present in Xenarthra. PLoS Genet 2006, 2(7):el09.

19. Yang F, Graphodatsky AS, Li T, Fu B, Dobigny G, Wang J, Perelman PL, Serdukova NA, Su W, O'Brien PC, Wang Y, Ferguson-Smith MA, Volobouev $\mathrm{V}$, Nie W: Comparative genome maps of the pangolin, hedgehog, sloth, anteater and human revealed by cross-species chromosome painting: further insight into the ancestral karyotype and genome evolution of eutherian mammals. Chromosome Res 2006, I4(3):283-296.

20. Graphodatsky AS, Yang F, Perelman PL, O'Brien PC, Serdukova NA Milne BS, Biltueva LS, Fu B, Vorobieva NV, Kawada SI, Robinson TJ, Ferguson-Smith MA: Comparative molecular cytogenetic studies in the order Carnivora: mapping chromosomal rearrangements onto the phylogenetic tree. Cytogenet Genome Res 2002, 96(I4): $137-145$

21. Nie W, Wang J, O'Brien PC, Fu B, Ying T, Ferguson-Smith MA, Yang F: The genome phylogeny of domestic cat, red panda and five mustelid species revealed by comparative chromosome painting and G-banding. Chromosome Res 2002, I 0(3):209-222.
22. Ye J, Biltueva L, Huang L, Nie W, Wang J, Jing M, Su W, Vorobieva NV, liang $X$, Graphodatsky AS, Yang F: Cross-species chromosome painting unveils cytogenetic signatures for the Eulipotyphla and evidence for the polyphyly of Insectivora. Chromosome Res 2006, I4(2): I5I-159.

23. McKenna MC: Toward a phylogenetic classification of the Mammalia. In Phylogeny of the primates Edited by: Luckett WP, Szalay FS. New York, Plenum; 1975:21-46.

24. Amrine-Madsen H, Koepfli KP, Wayne RK, Springer MS: A new phylogenetic marker, apolipoprotein B, provides compelling evidence for eutherian relationships. Mol Phylogenet Evol 2003, 28(2):225-240.

25. Murphy WJ, Eizirik E, Johnson WE, Zhang YP, Ryder OA, O'Brien S]: Molecular phylogenetics and the origins of placental mammals. Nature 2001, 409(6820):614-618.

26. Waddell PJ, Shelley S: Evaluating placental inter-ordinal phylogenies with novel sequences including RAG I, gamma-fibrinogen, ND6, and mt-tRNA, plus MCMC-driven nucleotide, amino acid, and codon models. Mol Phylogenet Evol 2003, 28(2): 197-224.

27. Liu FG, Miyamoto MM: Phylogenetic assessment of molecular and morphological data for eutherian mammals. Syst Biol 1999 , 48(I):54-64

28. Springer MS, Amrine HM, Burk A, Stanhope MJ: Additional support for Afrotheria and Paenungulata, the performance of mitochondrial versus nuclear genes, and the impact of data partitions with heterogeneous base composition. Syst Biol 1999, 48(I):65-75

29. Kullberg M, Nilsson MA, Arnason U, Harley EH, Janke A: Housekeeping genes for phylogenetic analysis of eutherian relationships. Mol Biol Evol 2006, 23(8): |493-1503.

30. Delsuc F, Vizcaino SF, Douzery EJ: Influence of Tertiary paleoenvironmental changes on the diversification of South American mammals: a relaxed molecular clock study within xenarthrans. BMC Evol Biol 2004, 4: I I.

31. Eizirik E, Murphy WJ, O'Brien SJ: Molecular dating and biogeography of the early placental mammal radiation. J Hered 2001, 92(2):212-219.

32. Springer MS, Stanhope MJ, Madsen O, de Jong WW: Molecules consolidate the placental mammal tree. Trends Ecol Evol 2004, I 9(8):430-438.

33. Liu FG, Miyamoto MM, Freire NP, Ong PQ, Tennant MR, Young TS, Gugel KF: Molecular and morphological supertrees for eutherian (placental) mammals. Science 200I, 291 (5509): I 786-I789.

34. Douady CJ, Catzeflis F, Kao DJ, Springer MS, Stanhope M]: Molecular evidence for the monophyly of tenrecidae (mammalia) and the timing of the colonization of Madagascar by Malagasy Tenrecs. Mol Phylogenet Evol 2002, 22(3):357-363.

35. Douady CJ, Chatelier PI, Madsen O, de Jong WW, Catzeflis F, Springer MS, Stanhope MI: Molecular phylogenetic evidence confirming the Eulipotyphla concept and in support of hedgehogs as the sister group to shrews. Mol Phylogenet Evol 2002, 25(I):200-209.

36. Kriegs JO, Churakov G, Kiefmann M, Jordan U, Brosius J, Schmitz J: Retroposed elements as archives for the evolutionary history of placental mammals. PLoS Biol 2006, 4(4):e9l.

37. Nishihara H, Satta Y, Nikaido M, Thewissen JG, Stanhope MJ, Okada N: A retroposon analysis of Afrotherian phylogeny. Mol Biol Evol 2005, 22(9): 1823-1833.

38. Stanyon R, Yang F, Cavagna P, O'Brien PC, Bagga M, Ferguson-Smith $M A$, Wienberg J: Reciprocal chromosome painting shows that genomic rearrangement between rat and mouse proceeds ten times faster than between humans and cats. Cytogenet Cell Genet 1999, 84(3-4): I50-155.

39. Telenius H, Carter NP, Bebb CE, Nordenskjold M, Ponder BA, Tunnacliffe A: Degenerate oligonucleotide-primed PCR: genera amplification of target DNA by a single degenerate primer. Genomics 1992, I3(3):718-725.

40. Froenicke L, Caldes MG, Graphodatsky A, Muller S, Lyons LA, Robinson T], Volleth M, Yang F, Wienberg J: Are molecular cytogenetics and bioinformatics suggesting diverging models of ancestral mammalian genomes? Genome Res 2006, I 6(3):306-310. 